\title{
THE NOAA GOES-12 SOLAR X-RAY IMAGER (SXI) 1. INSTRUMENT, OPERATIONS, AND DATA
}

\author{
S. M. HILL ${ }^{1}$, V. J. PIZZO ${ }^{1}$, C. C. BALCH ${ }^{1}$, D. A. BIESECKER ${ }^{1}$, P. BORNMANN ${ }^{1, *}$, \\ E. HILDNER ${ }^{1}$, L. D. LEWIS ${ }^{1}$, R.N. GRUBB ${ }^{1}$, M.P. HUSLER ${ }^{1}$, K. PRENDERGAST ${ }^{1}$, \\ J. VICKROY ${ }^{1}$, S. GREER ${ }^{1}$, T. DEFOOR ${ }^{1}$, D. C. WILKINSON ${ }^{2}$, R. HOOKER $^{3}$, \\ P. MULLIGAN ${ }^{3}$, E. CHIPMAN ${ }^{4}$, H. BYSAL ${ }^{4}$, J. P. DOUGLAS ${ }^{4}$, R. REYNOLDS $^{4}$, \\ J. M. DAVIS ${ }^{5}$, K. S. WALLACE ${ }^{5}$, K. RUSSELL ${ }^{5}$, K. FREESTONE $^{5}$, D. BAGDIGIAN ${ }^{5}$,

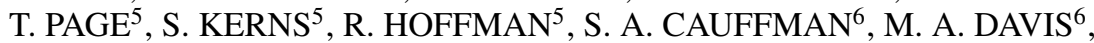 \\ R. STUDER $^{6}$, F. E. BERTHIAUME 6 , T. T. SAHA ${ }^{6}$, G. D. BERTHIUME ${ }^{7}$, \\ H. FARTHING ${ }^{8}$ and F. ZIMMERMANN ${ }^{9, * *}$ \\ ${ }^{1}$ NOAA Space Environment Center, 325 Broadway, Boulder, CO 80305, U.S.A. \\ (e-mail: steven.hill@noaa.gov) \\ ${ }^{2}$ NOAA National Geophysical Data Center, 325 Broadway, Boulder, CO 80305, U.S.A. \\ ${ }^{3}$ NOAA NESDIS, Silver Spring, MD 20910, U.S.A. \\ ${ }^{4}$ NOAA NESDIS/SOCC, Suitland, MD 20746, U.S.A. \\ ${ }^{5}$ NASA Marshall Space Flight Center, Huntsville, AL 35812, U.S.A. \\ ${ }^{6}$ NASA Goddard Space Flight Center, Greenbelt, MD 20771, U.S.A. \\ ${ }^{7}$ MIT Lincoln Laboratories, Lexington, MA 02420, U.S.A. \\ ${ }^{8}$ Swales Aerospace, Beltsuille, MD, 20705, U.S.A. \\ ${ }^{9}$ Space Systems Loral, Palo Alto, CA, 9430, U.S.A. \\ *Present address: Ball Aerospace \& Technologies Corp., 1600 Commerce Street, \\ Boulder, CO 80306-1062, U.S.A. \\ ** Present address: Swales Aerospace, Beltsville, MD, 20705, U.S.A.
}

(Received 27 August 2004; accepted 29 November 2004)

\begin{abstract}
The Solar X-ray Imager (SXI) was launched 23 July 2001 on NOAA's GOES-12 satellite and completed post-launch testing 20 December 2001. Beginning 22 January 2003 it has provided nearly uninterrupted, full-disk, soft X-ray solar images, with a continuous frame rate significantly exceeding that for previous similar instruments. The SXI provides images with a 1 min cadence and a single-image (adjustable) dynamic range near 100. A set of metallic thin-film filters provides temperature discrimination in the $0.6-6.0 \mathrm{~nm}$ bandpass. The spatial resolution of approximately 10 arcsec FWHM is sampled with 5 arcsec pixels. Three instrument degradations have occurred since launch, two affecting entrance filters and one affecting the detector high-voltage system. This work presents the SXI instrument, its operations, and its data processing, including the impacts of the instrument degradations. A companion paper (Pizzo et al., this issue) presents SXI performance prior to an instrument degradation that occurred on 5 November 2003 and thus applies to more than 420000 soft X-ray images of the Sun.
\end{abstract}

\section{Introduction}

The solar origin of disturbances to the space environment, or space weather, is well recognized. Space weather affects a broad range of technological endeavors on a daily basis. For instance, power companies (independent system operators) limit transmission capacity and generation based on geomagnetic activity, and airlines 
consider aircrew radiation dosage on polar routes from the United States to the Far East. One charge of NOAA's Space Environment Center (SEC) is to operationally specify ("nowcast") and forecast space environment conditions. Since all space weather originates with the Sun, it is a primary objective of SEC's observations.

With the launch of the Solar X-ray Imager (SXI) on Geosynchronous Operational Environmental Satellite 12 (GOES-12) on 23 July 2001, the first operational (used here to indicate missions dedicated to real-time space environment specification) observation capability for the Sun's X-ray corona began. GOES-12 was in on-orbit storage until "called up" for operations 22 January 2003. This work provides a description of the SXI, its operation, and its data processing. It includes discussion of the operational impacts of three system degradations that have occurred since launch: pinhole degradation of one or more entrance filters, degradation of the highvoltage power supply (HVPS), and complete failure of one of the entrance filters. A companion paper (Pizzo et al., this issue, henceforth Paper 2) provides information on SXI's calibration and performance prior to the entrance filter complete failure.

The scientific community has realized the great potential for improved understanding of the solar corona using X-ray imagery for three decades, beginning with sub-orbital rocket flights in the 1960s (Vaiana, Krieger, and Timothy, 1973). Significant contributions to this understanding have been made in particular by the Orbiting Solar Observatory (OSO) spacecraft series (Krieger et al., 1972), the Skylab S-054 (Vaiana and Rosner, 1978) and S-056 (Underwood and McKenzie, 1978) telescopes and by the Yohkoh soft X-ray telescope (SXT) (Martens and Cauffman, 2002; Tsuneta et al., 1991; Watanabe, Kosugi, and Sterling, 1998). Early recognition by NOAA of the importance of solar X-ray imaging led to a proposal for an operational imager in 1967 and ground prototype development in the early 1980s (Cessna et al., 1983). The GOES-12 SXI represents the culmination of NOAA and USAF efforts as the first in a series of solar X-ray imagers to fly on GOES spacecraft.

It is important to note that there are clear differences between NOAA's forecastdriven observational requirements and those of a purely research-oriented instrument. Whereas researchers may seek to 'push the envelope' on instrument resolution or sensitivity, NOAA values reliability and continuity of coverage in its operational systems. NOAA's Geosynchronous Operational Environmental Satellite program has included space environment measurements since its inception with the launch of the first Synchronous Meteorological Satellite (SMS-1) in 1974. These measurements have provided a consistent historical record and have grown in importance as the Nation's reliance on space- and ground-based technology affected by the space environment accelerates. The GOES-12 SXI will now add to this tradition and continue the historical record of soft X-ray imaging of the Sun begun by OSO and Skylab and epitomized by Yohkoh's SXT.

The NOAA Space Weather Scales (Poppe, 2000) illustrate the primary space weather processes of interest to NOAA: geomagnetic storms, solar radiation storms, and radio blackouts. Technologies affected by geomagnetic storms include terrestrial power transmission grids and spacecraft attitude control systems. Solar 
radiation storms increase the radiation dose to astronauts and high-latitude/highaltitude aircrews and affect spacecraft electronics. Radio blackouts result from 'short-wave fade' as the ionosphere responds to solar X-ray radiation.

Behind each of these impacts is a solar driver. Geomagnetic storms can be initiated by one of two solar sources. Coronal holes - large, long-lived areas of open magnetic field in the solar atmosphere - are the sources of high-speed solar wind that can drive recurring geomagnetic storms. The largest geomagnetic storms occur when a coronal mass ejection (CME) from the Sun throws into the interplanetary medium billions of tons of plasma, a portion of which envelops Earth's magnetic field. Solar radiation storms originate either in direct association with solar flares and with shocks associated with CMEs. Radio blackouts occur in direct response to the enhanced X-ray radiation from solar flares. In addition, increased satellite drag is associated with longer-term increases in solar EUV flux. These solar drivers lead to specific high-level forecast goals for SXI observations:

- Locate coronal holes for forecasts of recurring geomagnetic activity,

- Locate flares for forecasts of solar energetic particle events,

- Assess active region complexity for flare forecasts,

- Monitor active regions beyond the east limb for solar activity $\left(\mathrm{f}_{10.7}\right)$ forecasts, and

- Determine occurrence of CMEs.

Instrument performance requirements are derived by evaluating the characteristics of the solar phenomena related to the forecast goal. These requirements are defined in terms of instrument response, temperature discrimination, spatial resolution, and image cadence. Solar signatures of potentially geoeffective phenomena are listed in Table I along with their relevant observational properties.

TABLE I

Solar signature summary in soft X-rays.

\begin{tabular}{|c|c|c|c|c|c|}
\hline Feature & $\begin{array}{l}\text { Temperature } \\
\text { (MK) }\end{array}$ & $\begin{array}{l}\log \mathrm{EM} \\
\int n_{e}^{2} \mathrm{~d} l \\
\left(\mathrm{~cm}^{-5}\right)\end{array}$ & $\begin{array}{l}\text { Flux at } \operatorname{Earth}^{\mathrm{a}} \text { (photon } \\
\mathrm{cm}^{-2} \operatorname{arcsec}^{-1} \mathrm{~s}^{-1} \text { ) }\end{array}$ & $\begin{array}{l}\text { Temporal } \\
\text { scale }\end{array}$ & $\begin{array}{l}\text { Spatial } \\
\text { scale } \\
\left(R_{\text {SUN }}\right)\end{array}$ \\
\hline Coronal holes & $0.9-2.4$ & $25.5-27.0$ & $0.3-26$ & Weeks-months & $0.05-0.5$ \\
\hline Flares & $7-20$ & $30-32$ & $19000-1000000$ & Seconds-hours & $0.01-0.2$ \\
\hline Active regions & $2-3$ & $28-29$ & $230-2800$ & Days-weeks & $0.05-0.2$ \\
\hline Solar activity & $0.9-3.0$ & $26-29$ & $1.0-2800$ & Hours-days & $0.05-0.2$ \\
\hline \multicolumn{6}{|l|}{ CMEs } \\
\hline Flare arcades & $7-20$ & $30-32$ & $19000-1000000$ & Minutes-hours & $0.1-0.2$ \\
\hline Other $^{b}$ & - & - & - & Seconds-minutes & $0.1-0.5$ \\
\hline
\end{tabular}

${ }^{a}$ Flux at Earth is computed using an isothermal plasma emission model (Mewe, Gronenschild, and Oord, 1985) with a standard composition and a 0.6-6.0 nm bandpass.

b Other transients indicating CMEs include 'ejecta', waves, and dimmings. They have a wide range of temperatures, emission measures, and temporal scales. 
This paper describes the GOES-12 SXI instrument, its operations, and its data processing. Section 2 discusses the spacecraft configuration and the SXI instrument accommodation. Section 3 describes the instrument itself. Section 4 presents the instrument operations concept and a chronology of instrument status. Section 5 describes data processing, products, and dissemination. Section 6 concludes by highlighting the role of this instrument in the current context of solar research.

\section{GOES}

The mission of the GOES spacecraft is to obtain observations of Earth's weather and space environment (GOES I-M Data Book, 1996). The baseline constellation consists of two operational satellites and one satellite stored on-orbit. The operational satellites are deployed in a geosynchronous orbit $6.6 R_{\mathrm{E}}$ from Earth's center at $75^{\circ}$ and $135^{\circ}$ west longitudes. The Earth Imager and Sounder are the primary instruments. Call-up of the stored satellite is dependent on a loss of meteorological data from these instruments. GOES is also equipped with the Space Environment Monitor (SEM) (GOES I-M Data Book, 1996), composed of particle sensors (Onsager et al., 1996), a magnetometer (Singer et al., 1996), a solar disk-integrated X-ray sensor (XRS) (Bornmann et al., 1996a), and an SXI.

The accommodation of the SXI on GOES had to be accomplished without impact to the performance of the spacecraft in its Earth imaging role. Unlike the case of a solar viewing scientific instrument where the spacecraft is designed to meet the pointing requirements of the instrument, the GOES SXI had to be accommodated within the framework of a basically Earth viewing platform. To facilitate this, a decision was made to place a Sun sensor that provides precision pointing knowledge for the SXI within the instrument itself and to accept a lower accuracy in solar pointing from the spacecraft than would normally be expected for a scientific imaging instrument. The compromises necessary to accomplish this are offset for operational purposes, however, by the economy of integration with a major NOAA mission, and the advantage presented by a geostationary platform for real-time operation and data transmission.

In the transition from the earlier spinning GOES spacecraft to the three-axesstabilized platforms of the GOES I-M series, the solar array pointing had already been utilized to accommodate the XRS instrument on the solar array yoke. (GOES spacecraft receive letter designations prior to on-orbit delivery at which time numbers are assigned, e.g., the GOES I-M spacecraft became GOES 812.) After the basic spacecraft design had been finalized, the contractor, Loral Aerospace, was asked to study the feasibility of adding the SXI to the solar-pointed instrument complement. The results of this study suggested that an instrument that would meet the basic operational requirement could be accommodated on the solar array yoke. However, significant modifications to the spacecraft to provide more accurate pointing and reduce the jitter caused by oscillations in the solar array 


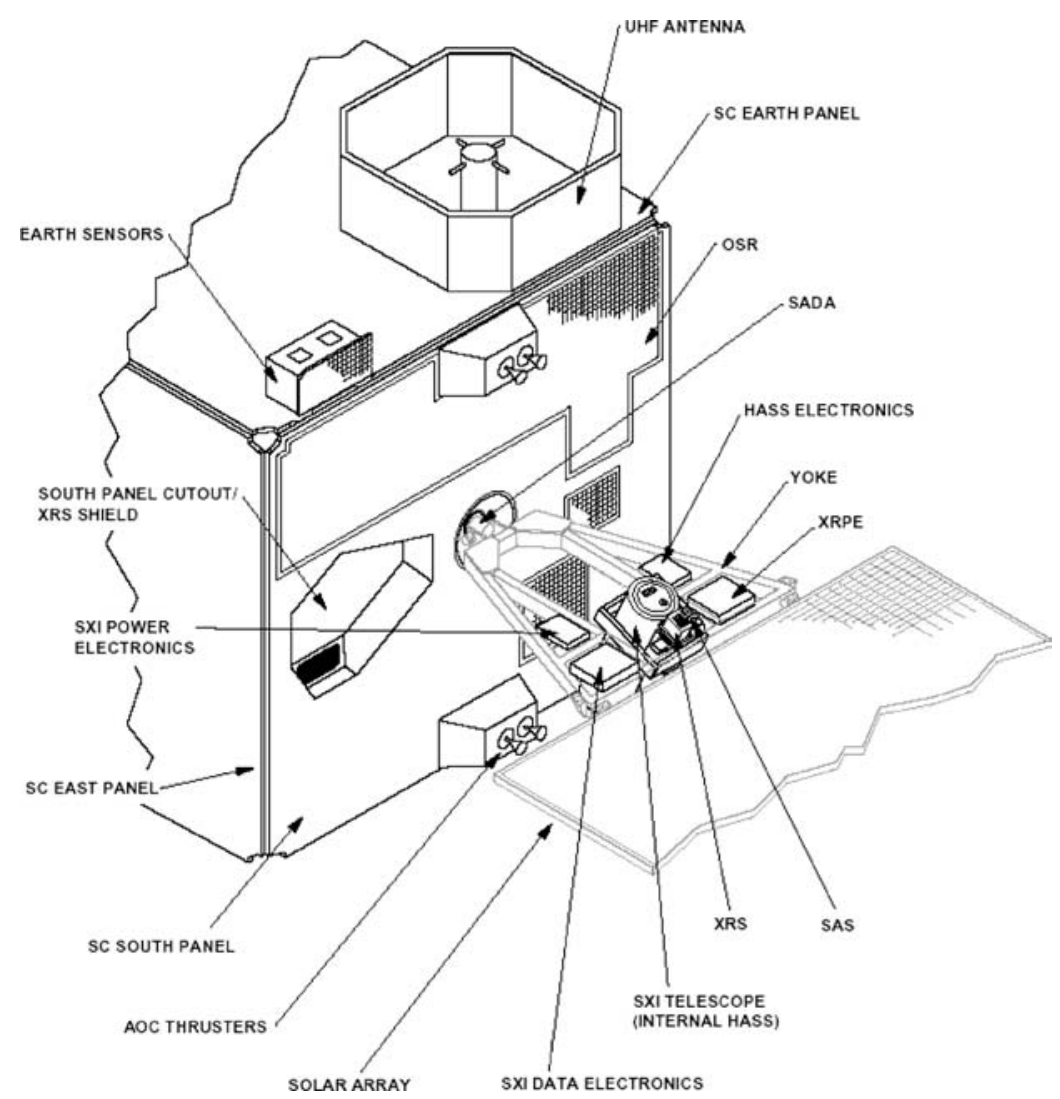

Figure 1. Instrument mounting configuration (GOES I-M Data Book (1996)).

would be necessary (Zimmermann, Zwirn, and Davis, 2004). In addition, significant constraints were placed on the SXI instrument dimensions and weight that could be accommodated.

The SXI and other solar instruments are mounted on a pointing platform on the yoke of the solar array panel because GOES spacecraft are three-axis oriented to the nadir (Figure 1). Seasonal north-south variations in the solar array pointing are corrected with the X-Ray Positioner (XRP), a single-axis gimbal platform. The $\mathrm{XRP}$ is attached to the solar array yoke. The Solar Array Drive Assembly (SADA) adjusts the east-west pointing of the yoke at an average rate of $15 \mathrm{arcsec} / \mathrm{s}$. The rotation rate of the SADA is achieved via a stepper motor that nominally takes one 24 arcsec step every $1.6 \mathrm{~s}$. The XRP operates with a closed loop controller driven by the Sun Analog Sensor (SAS) to maintain Sun pointing. However, because the XRP and SADA do not remove all orbital and spacecraft attitude effects on pointing, the solar image drifts somewhat in the SXI field of view. Both XRP and SADA pointing can be adjusted via ground command. Typical pointing errors of \pm 3 arcmin are achieved over a period of $24 \mathrm{~h}$. 
TABLE II

Selected spacecraft and mission properties (GOES I-M Data Book (1996)).

\begin{tabular}{ll}
\hline Parameter & Value \\
\hline Deployment mass & $2270 \mathrm{~kg}$ \\
Maximum dimension & $26.9 \mathrm{~m}$ \\
Maximum power & $1.3 \mathrm{~kW}$ \\
Spacecraft mission design life & 5 years \\
Launch date & 23 July 2001 \\
Formal operational date (SXI) & 26 April 2003 \\
Multiuse data link (MDL) rate & $100 \mathrm{kbps}$ \\
Imaging stability (meteorology) & $<15$ arcsec over $15 \mathrm{~min}$ \\
\hline
\end{tabular}

SXI exposures are taken between steps of the SADA. Since the spacecraft rotates at $15 \operatorname{arcsec~s} \mathrm{s}^{-1}$ about the celestial pole to maintain nadir pointing, solar image motion across the detector during an exposure must be compensated. This is accomplished by electronically advancing detector array lines at the proper rate. In addition, the solar array particularly, and the spacecraft in general, are susceptible to jitter. Further discussion of performance regarding pointing control, knowledge, and jitter is provided in Paper 2.

Image and most housekeeping data from the SXI are routed to the ground via the S-band Multiuse Data Link (MDL) at $100 \mathrm{kbps}$. The data are downlinked as transfer frames in close compliance with the Consultative Committee for Space Data Systems (CCSDS) format. Images are downlinked as soon as they are taken, which allows for very accurate time tagging of the data by the ground processing system. NOAA's SEC receives the data in real-time (data transmission latency only) and assembles it into data products in real-time for distribution to forecasters and other users. Further information on time-tagging accuracy is presented in Paper 2.

The GOES-12 is deployed as the operational 'East' satellite stationed above the equator at longitude $75^{\circ}$ West. Its geosynchronous orbit provides nearly continuous solar viewing, with data drop-outs of less than 90 min occurring daily during two 40 day eclipse seasons each year. The spacecraft is designed for up to 2 years of on-orbit storage followed by 5 years of active operating life. The SXI flying on GOES-12 is designated as a prototype with an operating design life of 3 years after on-orbit storage. Further information on the GOES-12 spacecraft and mission is outlined in Table II.

\section{Instrument Description}

The GOES-12 SXI was built by NASA's Marshall Space Flight Center (MSFC) with funding from the US Air Force (for background information see also Bornmann 


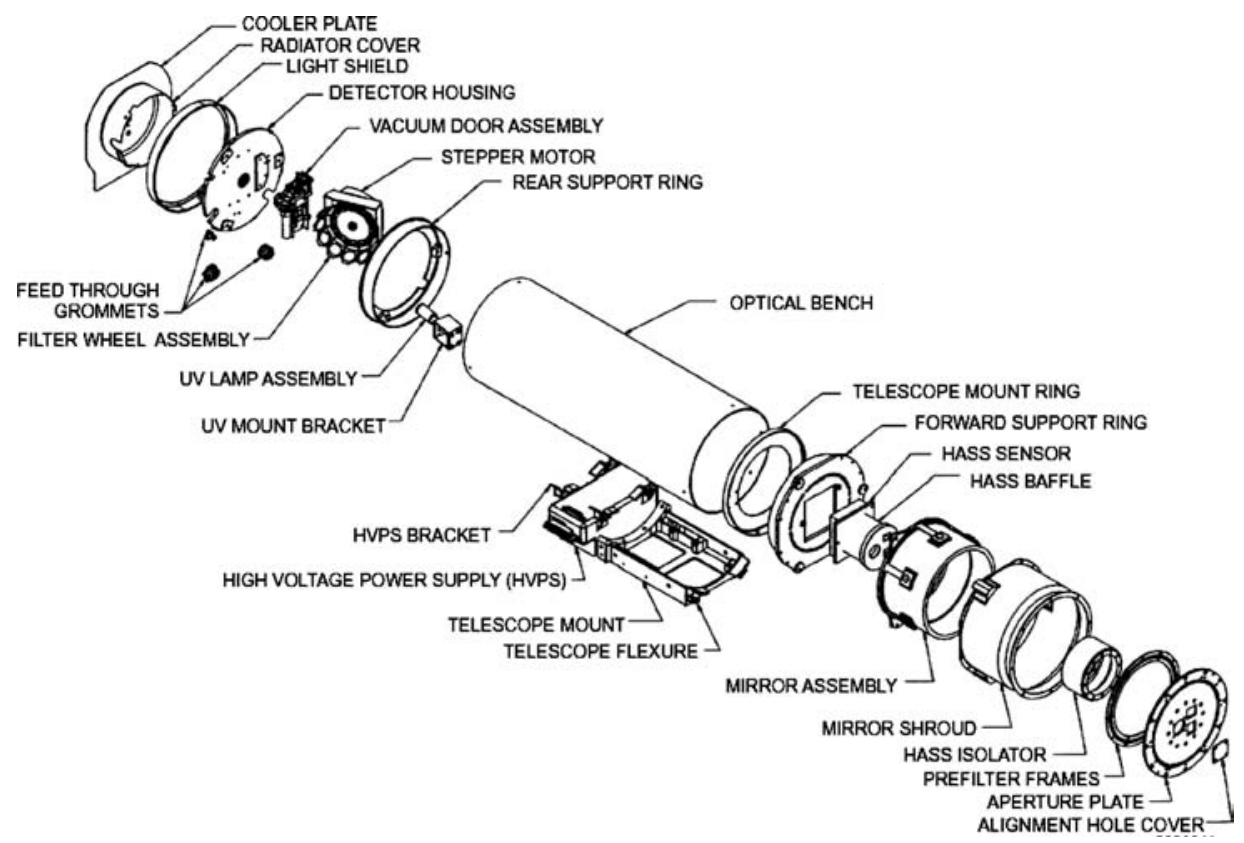

Figure 2. Instrument exploded view (GOES I-M Data Book (1996)).

et al., 1996b; Davis et al., 1994; GOES I-M Data Book, 1996). The telescope assembly is shown schematically in Figure 2. The SXI uses a classic Wolter I grazing incidence optical design with a nickel-coated, monolithic Zerodur mirror (Smithers and Zissa, 1996) to support an overall instrument 0.6-6.0 nm bandpass. Metallic thin-film entrance filters reject IR-visible-UV solar radiation. A set of selectable thin-film filters allows for rudimentary plasma temperature discrimination. The detector is comprised of three components: A microchannel plate (MCP) intensifier, a fiber optic taper (FOT), and a visible light CCD (Corder, 1994; Russell, 1996). The MCP acts like an array of electron-multiplier tubes with a gain of $10^{3}-10^{5}$, depending on applied voltage. The resulting electron clouds impact a phosphor plate that emits at visible wavelengths. The phosphor is optically linked via the FOT to the $512 \times 512$ pixel CCD. The rationale for using this design included: very good visible light rejection, very good 'long-wave' $(2-10 \mathrm{~nm}) \mathrm{X}$-ray sensitivity, and the lack of CCDs sensitive to longer wavelength X-rays at the time of design. An ultraviolet illumination source is included for detector "aliveness" verification. Operational control of imaging sequences is driven by a set of on-board tables that can be modified by ground command (Wallace and Freestone, 2001; Wallace, Brown, and Freestone, 1998). The instrument is generally operated at a basic 1-min image cadence. A High-Accuracy Sun Sensor (HASS) integrated into the instrument provides pointing knowledge.

Many of the references mentioned earlier have provided partial or comprehensive descriptions of the SXI at various stages throughout its development and ground 
test. The objective of this section is to synthesize a current, definitive description of the SXI with particular relevance to researchers and other data users.

\subsection{OPTICS}

An outline of the SXI optical system, including the optical bench, aperture, prefilters, and analysis filters is given. Detailed performance of these components and the overall system is provided in Paper 2. Basic system properties are shown in Table III.

The optical bench provides structural integrity and maintains the separation between the mirror and the focal plane subassembly to $\pm 10 \mu \mathrm{m}$ over a temperature range of $\pm 40{ }^{\circ} \mathrm{C}$. The bench was laid up by hand using sheets of carbon fibers impregnated with a cyanate ester resin. The bench material is highly hydrophobic and thermally stable, thereby minimizing water vapor outgassing and possible condensation on the detector array.

Six identical arc-shaped pre-filters manufactured by Luxel Corp. are mounted on the front aperture plate of the telescope. These filters prevent solar ultraviolet, visible, and infrared radiation from entering the interior of the telescope. Each pre-filter is a sandwich of titanium, polyimide, and aluminum. The inner layer is $100 \mathrm{~nm}$ of aluminum. The outer titanium layer blocks the aluminum transmission window between 20 and $80 \mathrm{~nm}$ and thus suppresses the very strong He II 30.4 $\mathrm{nm}$ upper chromospheric line. In addition, the visible and infrared properties of titanium allow the entrance filters to operate at a lower temperature under the solar heat load (Powell et al., 1997). The middle polyimide layer provides the strength needed to survive the acoustic launch loads. It is in these filters that a pinhole light leak is believed to have formed between 20 December 2001 and 22 January 2003. In addition, it is believed that one of these filters completely failed on 5 November 2003. These events and their impacts on operations and data processing

TABLE III

Optical system properties (Wallace and Freestone, 2001).

\begin{tabular}{ll}
\hline Optical design & $\begin{array}{l}\text { Paraboloid-hyperboloid } \\
\text { (Wolter I) }\end{array}$ \\
\hline Radius at principle plane & $8.0 \mathrm{~cm}$ \\
Graze angle & $1.754^{\circ}$ \\
Axial mirror element length & $4.75 \mathrm{~cm}$ \\
Separation of principal and focal planes & $65.0 \mathrm{~cm}$ \\
Mirror microroughness & $5 \AA$ rms \\
Mirror material & Zerodur \\
Mirror surface coating & Nickel \\
Geometrical area & $7.3 \mathrm{~cm}^{2}$ \\
\hline
\end{tabular}


are described in Sections 4 and 5. The pinhole failure impact on system performance is described in Paper 2.

The mirror consists of a Wolter Type I grazing incidence parabola-hyperbola configuration manufactured by United Technologies Optical Systems. Both optical surfaces are fabricated from a single Zerodur element. A nickel coating was chosen for the mirror to optimize response to longer wavelength photons, particularly in the range of 2-6 nm. Obscuration of the mirror clear aperture by the aperture plate amounts to $10 \%$.

A 12-position filter wheel, whose motor was manufactured by Aeroflex Corporation of Plainview, New York, holds six unique thin-film filters plus an 'open' position (with no filter) for solar observations. Three of the filter types are redundant, so that 10 of the 12 positions are taken up for solar observations. In addition, there is a radiation blocker, stainless steel with a thickness of $7.9 \mathrm{~mm}$, that allows for true dark frames to be taken and a UV-diffuser for use with an internal UV 'aliveness' source.

\subsection{DETECTOR AND CAMERA}

The focal plane design (Figure 3) incorporates three components: a MCP as the detecting element, a phosphor-coated FOT to modify the plate scale, and a chargecoupled device (CCD), sensitive to visible light, as the collecting element. NASA Marshall Space Flight Center led the design, development, and test of this detector stack (Corder, 1994).

The SXI's complex detection system has many benefits. The MCP blocks visible light and this requires less attenuation of visible light by filters which also attenuate

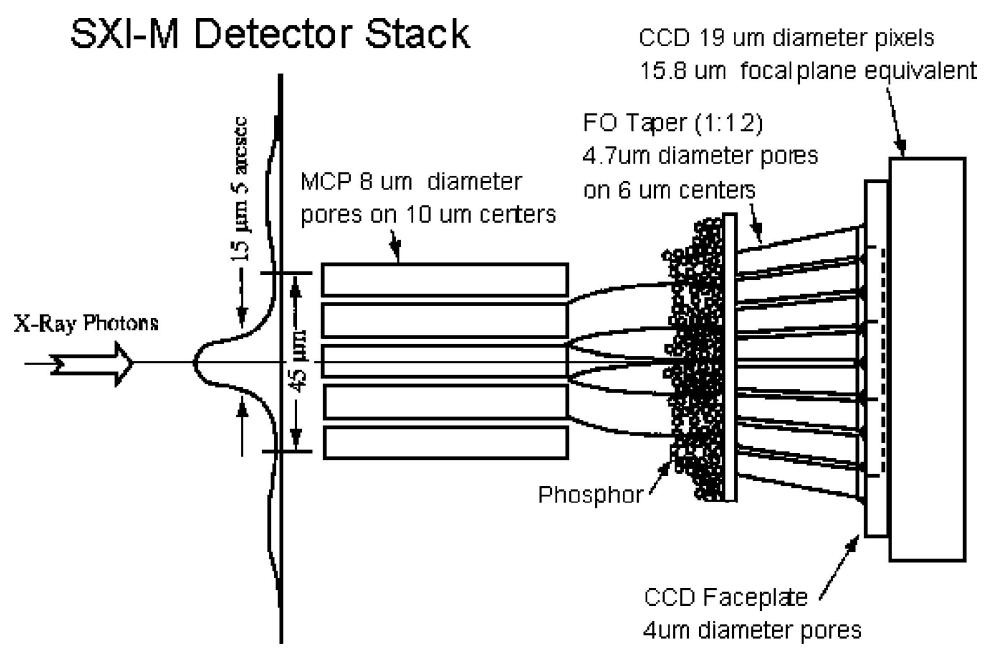

Figure 3. Detector stack schematic (Bornmann et al., 1997). 
low-energy soft X-rays of interest. In combination with the photon-counting nature of the detector, substantial improvements in sensitivity to emissions from plasmas below $2 \mathrm{MK}$ are achieved when compared to a bare $\mathrm{CCD}$. The detector configuration allows for adjustable gain that can be used in conjunction with exposure time to control signal-to-noise and dynamic range (see Paper 2). The design also provides electronic shuttering by controlling the accelerating voltage across the MCP. This voltage is turned on and off with rise times of $100 \mu$ s to accommodate exposure times as short as $1.5 \mathrm{~ms}$.

Galileo Electro-Optics Corporation (now owned by Burle Electronics) manufactured the "High-Output Technology" MCP that acts as an electron multiplier. The programmable gain voltage provides for a large dynamic range. The plate has $8 \mu \mathrm{m}$ diameter pores on $10 \mu \mathrm{m}$ centers. The pores are normal to the surface of the plate, but the focused X-rays strike the plate at a $7.0^{\circ}$ incidence angle. Each photon detected causes at least one electron to be emitted from the photocathode at the front surface of the MCP. A large potential (up to $1200 \mathrm{~V}$ ) across the MCP provides energy to the electron as it travels down a pore on its way to the phosphor. Each time the electron hits the wall of the pore, its energy causes the release of more electrons. This cascade results in the creation of 1000-10000 electrons per detected photon and follows a statistical distribution independent of the energy of the detected photon.

The electron avalanche from the MCP strikes the phosphor deposited on the face of the FOT. Proximity focusing of the electron cloud in a vacuum leads to wideangle scattering that is a function of the spacing between the MCP and the phosphor and their potential difference. This 'halo' effect is discussed further in Paper 2. The yttrium oxysulphide phosphor emits photons that match the peak sensitivity of the CCD in visible light. The FOT coupler provides a magnification of 1.2 that matches the plate scale of the focal plane to the CCD; i.e., the fiber pitch increases from 5 to $6 \mu$, changing the plate scale from 15.8 to $19 \mu$ so that one CCD pixel corresponds to 5.014 arcsec.

The TH7895A-(H) CCD is a $512 \times 512$ pixel device manufactured by Thomson Composets Militaires et Spatiaux of France. It has a full well depth of 450000 electrons. A practical dynamic range of order 1000 can be achieved by adjusting the gain of the MCP so that the average signal per photon is 150 electrons (dynamic range of 300 photons). With this 'tuning' the average signal per photon is well above the CCD's thermal noise level. Reference 'black' pixels are contained along a covered strip at the edge of the CCD, one for each CCD line. These pixels are not exposed to photons and are used by the amplifier to provide a reference thermal signal that is subtracted from the exposed pixels in that readout line. This design introduces a source of line-by-line noise to the data that is further discussed in Paper 2.

The layout of the solar image on the detector array is shown in Figure 4. The reference pixel row is depicted at the 'bottom' of the detector and the westward line advance (to compensate for spacecraft rotation) is shown. Also, note that the image 


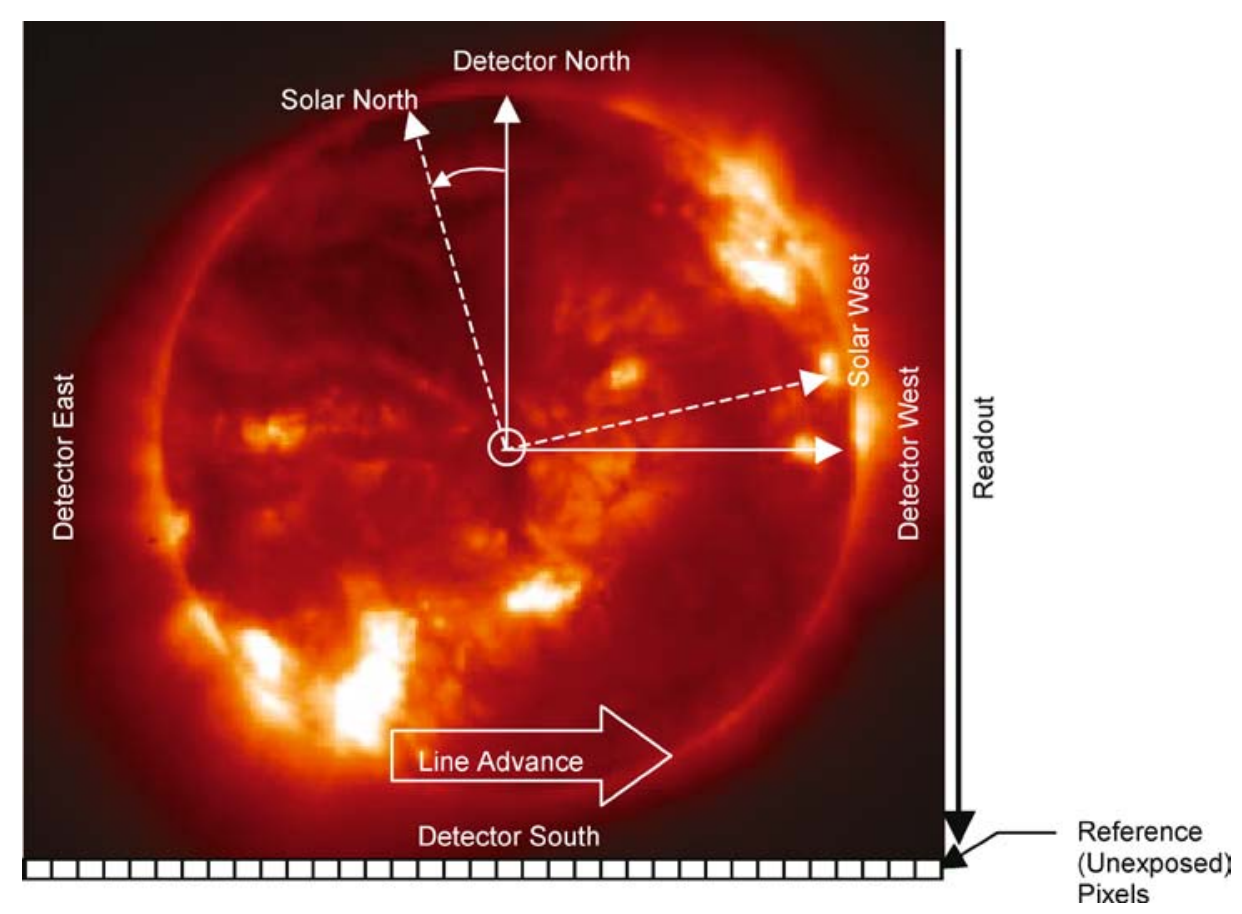

Figure 4. The first official SXI image of the Sun, taken on 2001 September 07, is shown in the context of the SXI detector array. Detector North is always within $0.5^{\circ}$ of celestial north. Line advance is from the left (east) to the right (west). Pixel readout is at the lower right.

of the Sun is rotated about the optical axis with respect to the detector. This occurs because the GOES-12 is an Earth-pointing spacecraft, so that the Sun goes through seasonal orientation changes similar to those seen by an Earth-bound observatory.

The detector can be illuminated with ultraviolet (UV) light by a small UV lamp (model 30-0075-01 from UVP, Inc., Upland, California) contained in the optical assembly. The MCP has only a small degree of sensitivity to the $254 \mathrm{~nm}$ mercury emission line. The lamp was primarily used for detector 'aliveness' testing during spacecraft integration and test, but it has also proven useful for limited flat-field testing.

\subsection{HIGH-ACCURACY SUN SENSOR}

The \pm 10 arcsec SXI pointing knowledge requirement is met by the Adcole Corporation (Marlborough, Massachusetts) HASS. This sensor provides the two coordinates of the Sun-center location relative to the SXI optical axis every $16 \mathrm{~ms}$ during an exposure. The HASS Sensor Head (HSH) is located within the center of the SXI telescope (roughly centered within the optical axis of the SXI mirror). It has a narrow $2^{\circ}$ field of view to avoid reflections from the spacecraft. The HSH is 
essentially a passive detector, composed of masks and photocells, which are read and processed by the HASS Electronics Box (HEB). The HEB receives the HSH outputs, and processes these signals into a 32-bit serial digital word. During image integration and HASS diagnostic mode, measurements from the HASS are taken with a frequency of one reading every $16 \mathrm{~ms}$. The least significant bit of the HASS corresponds to approximately 3.9 arcsec. The HASS system is used only to provide pointing knowledge during image integration. It provides no pointing control.

\subsection{ELECTRONICS AND CONTROL}

Three main electronics boxes provide for SXI electronics and control: The Data Electronics Box (DEB), Power Electronics Box (PEB), and the HEB, which was described earlier. The PEB receives spacecraft power over two lines, one for the instrument and one for instrument heaters. The PEB provides conditioned power at a variety of required voltages to the instrument, its electronics, its mechanisms, and its heaters. A total current monitor is provided.

Operation of the SXI is controlled through the DEB. An Analog Devices Inc. ADSP2100A digital signal processor operating at $2.0 \mathrm{MHz}$ serves as the central processing unit (CPU). This microprocessor runs the flight software and controls the overall activity of the instrument. Interface electronics receive uplinked commands and format output data for the spacecraft's MDL transmitter (described in Section 2). The DEB includes additional electronics to support other necessary functions for operating and monitoring the instrument.

Based on tables stored in read only memory (ROM) or uplinked into Random Access Memory (RAM) (see Section 4), the processor generates commands sent to the High Voltage Power Supply (HVPS) and to the CCD timing logic to begin an image-taking sequence. At the start of imaging a clock in the camera is reset. The clock automatically advances the CCD one column if the exposure exceeds 333 $\mathrm{ms}$, and at each integral multiple thereof, to compensate for orbital motion of the spacecraft of $15 \operatorname{arcsec~s}^{-1}$. This rate equates to a shift of one pixel every one-third of a second. Note that not only are the exposed pixels shifted, but the dark current reference pixels are also shifted.

The DEB provides reference voltages to the HVPS. The HVPS includes the timing logic and series regulators used to control the CCD and readout electronics. In addition, it consists of power supplies for the MCP (a programmable $1250 \mathrm{~V}$ supply) and for the phosphor layer (a programmable $5000 \mathrm{~V}$ power supply). The 1250 Volt power supply is required to have a $100 \mu \mathrm{s}$ rise/fall time, because the gating of this supply to the MCP effectively 'shutters' the image integration.

Once the exposure is complete, the CCD is read out at a $500 \mathrm{kHz}$ pixel rate and the reference 'black' pixels are subtracted from each line. This subtraction is referred to as 'line clamping.' The video signal then is transferred to one of two channels that provide either a linear or a logarithmic transfer function. The equivalent of a $4 \mathrm{DN}$ bias is then added to the signal to preserve noise characteristics by avoiding 
clipping of negative pixel values. The output of the selected channel is then passed to the input of the 10-bit analog/digital converter (ADC) in the DEB.

The first of two parity bits is generated over the 10-bit pixel value at the time of digitization and is stored in RAM along with the converted pixel values. Note that reference pixel values are neither stored nor downlinked. The second of the two parity bits is generated at the time the data are read from the RAM, and is generated over the 11-bit field that includes both the 10-bit pixel value and the 1-bit parity. This scheme allows any parity error to be identified as originating from a single event upset (SEU) in the memories, or an RF bit-flip during downlink.

The data are transmitted to the ground through the MDL interface with a 100 kbps capacity using a split phase data coding. The data are downlinked as transfer frames that are in close compliance with the Consultative Committee for Space Data Systems (CCSDS) format. These data are received in real time directly at NOAA's SEC in Boulder, Colorado. Details of ground processing and data products are given in Section 5.

\subsection{OPERATIONAL MODES AND WINDOWING}

The SXI system operates in five modes to accommodate imaging, testing, and basic survival. In Survival Mode, the SXI is unpowered except for thermostatically controlled heaters. Standby Mode is the SXI power-up default. The HVPS is inactive and no imaging is performed. Commands to perform diagnostics and housekeeping are allowed in this mode. Modifications to imaging tables are also allowed, and housekeeping is downlinked. Imaging Mode is the operational mode for the SXI. This mode may be entered only by ground command. In this mode, the SXI can image the Sun, take UV lamp images for diagnostic purposes, and take background images (radiation shield in place). The diagnostics mode is entered from Standby Mode in order to execute the Filter Wheel Diagnostics, MCP startup, XRP Oscillation Diagnostics, and RF Test Pattern. Safe Mode is entered either by ground command or by software if a serious hardware or commanding error is detected. When transitioning to Safe Mode, the HVPS is deactivated and the filter wheel is rotated to the radiation shield and disabled. The Safe Mode is used to prepare the instrument for a power loss and effects a graceful shutdown of the SXI subsystems.

In Imaging Mode, an option referred to as 'windowing' allows selected subarrays of data to be transmitted to the ground at a higher cadence than the full $512 \times 512$ array. When commanded into windowing, the SXI downlinks the pixel values from up to six ground-defined windows. The size of the windows can range from $16 \times 16$ pixels, up to the entire CCD.

\section{Operations}

Describing how an instrument is operated has two distinct facets. First, there is the instrument hardware and software and how it is controlled to perform its desired 
functions. Second, there is the description of 'mission operations', which includes such details as the organizations involved in planning, scheduling, and implementing operations. Additionally, for the SXI program, the term 'operational' also takes on a specific meaning.

For a typical research instrument, the term operational indicates that the initial on-orbit testing phase has been successfully completed and that data is being returned routinely. In NOAA, the term operational implies a number of qualities above and beyond those for a scientific instrument. The observations conducted by an operational instrument are time critical, repetitive rather than targeted, and must have high availability and reliability. The rationale for these criteria are that the data returned, along with derived products and forecasts, have a value for a variety of practical applications in addition to their intrinsic scientific value. For example, products and forecasts based on SXI data are of substantial economic importance to airline dispatchers, and are required to efficiently operate the power systems in the northeastern United States. Thus, NOAA and by extension, the SXI program, makes a commitment to continuously provide high-quality, calibrated data, in near real time to forecasters as well as the general public to enable value judgments to be made in a wide variety of contexts. It is this unique characteristic of being an operational instrument that drives much of the operations concept for SXI. This section first describes instrument operations, then mission operations, and finally provides a chronology of instrument status and observations.

\subsection{INSTRUMENT IMAGING OPERATIONS}

To meet the operational requirements of NOAA, the SXI image sequence control system uses a table-driven approach. Sequences of images are defined using structured loops with a high level of control over nesting, repetitions, and delays. These sequences are contained in the Image Control Tables (ICTs) and SXI hardware supports storage of 63 distinct ICTs. The number of sequences contained within an ICT is variable, but the maximum total number of entries (image IDs) in an ICT is 255. Each entry in an ICT references a set of imaging parameters via an index into the Exposure Setting Table (EST). SXI hardware supports 1023 entries in the EST. The EST itself does not contain the exposure parameters, but instead contains pointers to a set of six other parameter tables (PTs) that in turn contain the actual parameter values. The PTs consist of tables for the integration duration, the MCP gain, the analysis filter, the phosphor gain, the SADA step time offset, and the Special Features Field (SFF) - a bit field which acts as a set of discrete controls on specific imaging options.

Each of the tables may be stored in up to three memory areas: (1) ROM area with sequences or parameter values predefined at launch time, (2) a shadow RAM area where entries that were deemed most valuable prior to launch are copied from ROM to RAM upon system boot, and (3) an empty RAM area where totally new entries can be loaded via ground command. Using this approach, multiple sequences can be 
built and stored for later execution. The overall result is a flexible and autonomous SXI capable of meeting arbitrary imaging requirements and operating within ground commanding limitations.

In planning observing sequences, the key objectives for an operational system are: consistency, which allows forecasters to know what to expect; regularity, that is, a steady flow of images of given types at expected times; and meeting observational objectives, e.g. short exposures for unsaturated flare images and long exposures for coronal hole measurements. The dominant temporal components limiting the pace of sequence execution are image downlink time ( $\sim 40$ s for a full image), setup delay time (programmable, but defaults to $10 \mathrm{~s}$ - the driver is filter rotation), and solar array step synchronization (up to $3.28 \mathrm{~s}$ ). Normally, a sequence is designed to take images once per minute. While reduction in image size by windowing (Section 3.5) can result in a higher cadence, the effective maximum cadence for partial images is about four images per minute.

Governed by the guidelines mentioned earlier, the routine operations SXI observing sequence consists of nested, repeating blocks of images, slaved to UT time (Table IV). The entire program repeats every $6 \mathrm{~h}$. The basic block is the patrol block with four short-long exposure image pairs. Each pair is taken with the minimum time between exposures, e.g., approximately $40 \mathrm{~s}$. The first image of each pair is started on a 1 min clock boundary. This makes the spacing between the long image and the short image of the next pair approximately $80 \mathrm{~s}$. The spacing between image pair 'centers' is $2 \mathrm{~min}$. Short exposures are taken first so that the ground data system can substitute for saturated pixels in long-exposure images displayed in real time. The patrol block repeats every $8 \mathrm{~min}$ and generally has a higher cadence 'primary' patrol image that remains uninterrupted even when other 8 min block types are executed. To better facilitate forecaster selection of and access to images,

TABLE IV

SXI routine observation sequence prior to 5 November 2003.

\begin{tabular}{|c|c|c|c|c|c|c|c|c|c|c|c|}
\hline \multirow{2}{*}{$\begin{array}{l}\text { Start } \\
\text { (UT) }\end{array}$} & \multirow{2}{*}{$\begin{array}{l}\text { Total } \\
\text { time }\end{array}$} & \multirow{2}{*}{$\begin{array}{l}\text { Number of } \\
\text { repetitions }\end{array}$} & \multirow{2}{*}{$\begin{array}{l}\text { Block } \\
\text { type }\end{array}$} & \multicolumn{8}{|c|}{ Minute number } \\
\hline & & & & 1 & 2 & 3 & 4 & 5 & 6 & 7 & 8 \\
\hline 01:00 & 8 & 1 & DEM & $\mathrm{O}_{\mathrm{S}}$ & $\mathrm{O}_{\mathrm{L}}$ & $\mathrm{Pn}_{\mathrm{L}}$ & $\mathrm{Pk}_{\mathrm{L}}$ & $\mathrm{O}_{\mathrm{S}}$ & $\mathrm{O}_{\mathrm{L}}$ & $\mathrm{Bm}_{\mathrm{L}}$ & $\mathrm{Bk}_{\mathrm{L}}$ \\
\hline 01:09 & 112 & 14 & Patrol & $\mathrm{O}_{\mathrm{S}}$ & $\mathrm{O}_{\mathrm{L}}$ & $\mathrm{Pm}_{\mathrm{S}}$ & $\mathrm{Pm}_{\mathrm{L}}$ & $\mathrm{O}_{\mathrm{S}}$ & $\mathrm{O}_{\mathrm{L}}$ & $\mathrm{Bn}_{\mathrm{S}}$ & $\mathrm{Bn}_{\mathrm{L}}$ \\
\hline 03:00 & 8 & 1 & $\mathrm{Bkg} 1$ & $\mathrm{O}_{\mathrm{S}}$ & $\mathrm{O}_{\mathrm{L}}$ & $\mathrm{Bg}_{\mathrm{S}}$ & $\mathrm{Bg}_{\mathrm{L}}$ & $\mathrm{O}_{\mathrm{S}}$ & $\mathrm{O}_{\mathrm{L}}$ & $\mathrm{LL}_{\mathrm{S}}$ & $\mathrm{LL}_{\mathrm{L}}$ \\
\hline 03:09 & 112 & 14 & Patrol & $\mathrm{O}_{\mathrm{S}}$ & $\mathrm{O}_{\mathrm{L}}$ & $\mathrm{Pm}_{\mathrm{S}}$ & $\mathrm{Pm}_{\mathrm{L}}$ & $\mathrm{O}_{\mathrm{S}}$ & $\mathrm{O}_{\mathrm{L}}$ & $\mathrm{Bn}_{\mathrm{S}}$ & $\mathrm{Bn}_{\mathrm{L}}$ \\
\hline 05:00 & 8 & 1 & $\mathrm{Bkg} 2$ & $\mathrm{O}_{\mathrm{S}}$ & $\mathrm{O}_{\mathrm{L}}$ & $\mathrm{Bg}_{S}$ & $B g_{L}$ & $\mathrm{O}_{\mathrm{S}}$ & $\mathrm{O}_{\mathrm{L}}$ & $\mathrm{Bg}_{\mathrm{S}}$ & $\mathrm{Bg}_{\mathrm{L}}$ \\
\hline 05:09 & 112 & 14 & Patrol & $\mathrm{O}_{\mathrm{S}}$ & $\mathrm{O}_{\mathrm{L}}$ & $\mathrm{Pm}_{\mathrm{S}}$ & $\mathrm{Pm}_{\mathrm{L}}$ & $\mathrm{O}_{\mathrm{S}}$ & $\mathrm{O}_{\mathrm{L}}$ & $\mathrm{Bn}_{\mathrm{S}}$ & $\mathrm{Bn}_{\mathrm{L}}$ \\
\hline
\end{tabular}

Key: O, Open; $\mathrm{Pn}$, thin polyimide; $\mathrm{Pm}$, medium polyimide; $\mathrm{Pk}$, thick polyimide; $\mathrm{Bn}$, thin beryllium; $\mathrm{Bm}$, medium beryllium; Bk, thick beryllium; Bg, background (dark) frame; LL, light-leak background (Open filter). Subscripts: S, 'short' exposure ( $<1 \mathrm{~s})$; L, 'long' exposure (3 s). 
each image is assigned an intent category (coronal hole, active region, flare) based on its gain, exposure duration, and filter.

In the nominal operational sequence three filters are used for patrol: open, polyimide medium, and beryllium thin. These filters provide peak responses at 3.2, 4.0, and 5.0 MK, respectively. The Open image pair is repeated twice to obtain a cadence of 4 min per image pair. This cadence is uninterrupted in all image sequence blocks. The polyimide and beryllium filter pairs are repeated on an $8 \mathrm{~min}$ cadence. In other imaging sequence blocks, these two filter pairs are replaced with images for other purposes, e.g., dark-count backgrounds and open position light leak backgrounds.

The whole $6 \mathrm{~h}$ program begins with the so-called Differential Emission Measure (DEM) block. This block uses alternate polyimide and beryllium filters to provide full-disk, all-filter coverage of the Sun every $6 \mathrm{~h}$. While the DEM block itself is only $8 \mathrm{~min}$ long, full filter coverage is provided by polyimide medium and thin beryllium images taken from adjacent patrol blocks. This yields a 12 min total time to acquire images in all filters. The DEM block is synchronized with the SOHO EIT (Delaboudiniere et al., 1995) synoptic observing program in which images are taken in each of its four EUV filters at 01, 07, 13, and 19 UT. These similar observing programs complement one another by providing images of the Sun in a wide range of spectral bands four times per day.

The other two blocks are the background block and the test block. The background block substitutes a pair of dark current images and a pair of $0 \mathrm{~V}$ Open filter light leak images for the normal patrol block polyimide and beryllium image pairs. The test block is used for special operations including new background images, voltage sweeps, single photon tests, etc.

Finally, a special set of observations directed at the terrestrial thermosphere is used during the equinoctial eclipse periods. These observations are separate from the blocks described earlier and provide atmospheric attenuation data that can be used to estimate physical parameters in Earth's upper atmosphere such as density and temperature. While several variants of the routine operations observation sequence have been used, the two main variants, discussed in Section 4.3, account for approximately $80 \%$ of all observations.

\subsection{MISSION OPERATIONS}

Mission operations describes the organizational means by which an instrument achieves its objectives through the interaction of different responsible organizations in planning and implementing operations. A core group of four organizations bears the most responsibility for SXI and its data. Figure 5 shows the mission operations concept with the interrelationships between hardware, data flow, and organizations. NOAA's Satellite Operations Control Center (SOCC), in Suitland, Maryland, is responsible for generating and validating all command uploads, delivery of the command uploads to the spacecraft, telemetry verification of the command uploads, 


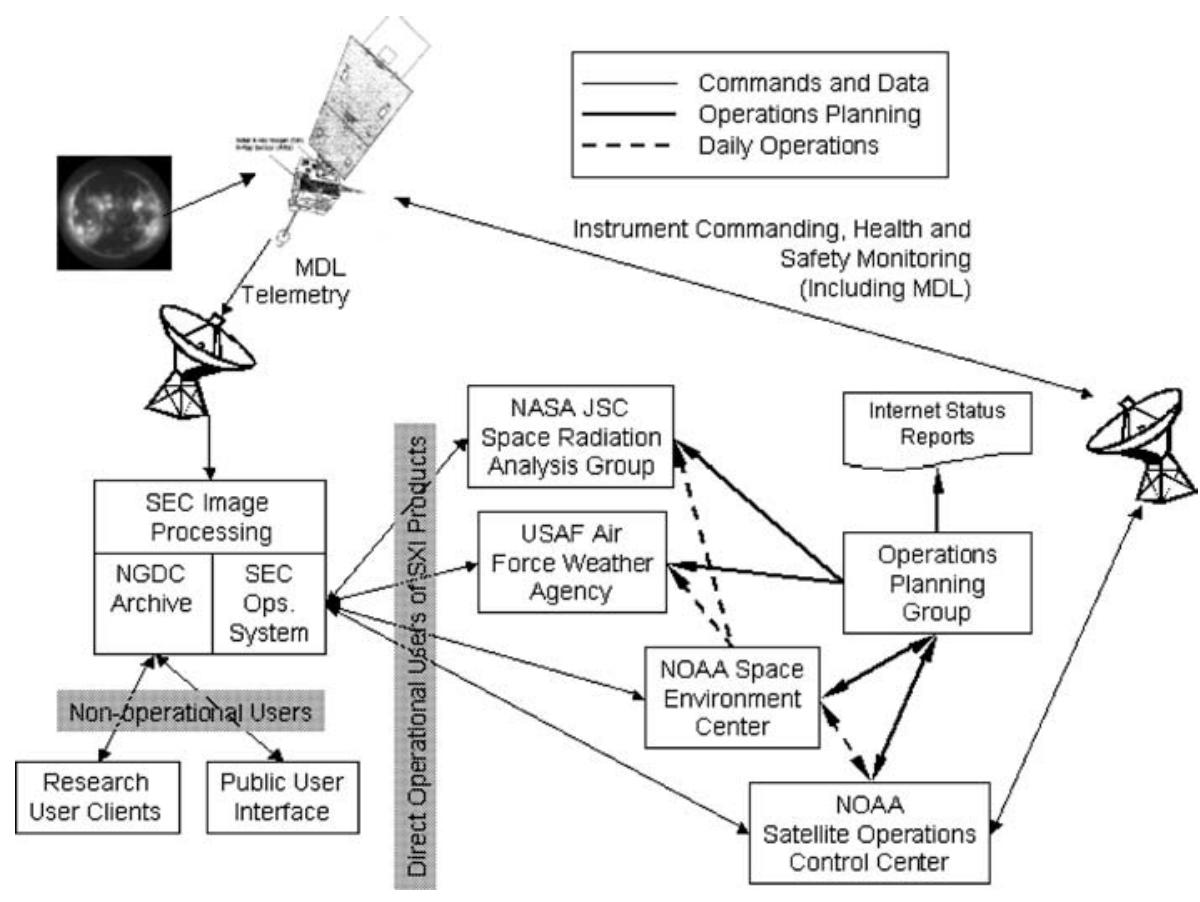

Figure 5. SXI operation concept.

and telemetry monitoring for health and safety. NOAA's SEC is responsible for observing program development and baseline management, data processing and dissemination, constructing and issuing space weather forecasts, warnings, and alerts, instrument calibration, and product development. Together, SEC and SOCC form the backbone of SXI operations.

Two primary direct data users fill out the core group of four organizations: SEC's sister organization in the USAF, the Air Force Weather Agency (AFWA), plays the same crucial space weather forecasting role for the DoD as SEC does for the civilian sector and NOAA's National Geophysical Data Center (NGDC) is the archivist of the data. In the context of SXI operations, USAF and AFWA chief responsibilities are to support observing program development and product development, as well as to provide inputs on operations scheduling. In the same context, NGDC primary responsibilities include providing customer feedback on SXI data to support SEC product development, performing additional product development, maintaining the data archive for and interface to the general public, and helping to maintain the near real-time data interface from SEC.

The joint Operations Planning Group (OPG) coordinates adjustments to instrument observing programs and periodic calibrations required by end-users of the data and has a membership that includes elements of SOCC and SEC. This group meets regularly via teleconference and communicates additionally via e-mail 
distribution lists. E-mail distribution lists are also used to provide near real-time operational notifications to support operations, e.g., a notice that the ground data system will be down for maintenance. As necessary, other groups such as NASA participate in the OPG, for example to assist in assessing instrument anomalies. By agreement, changes impacting the regular spacecraft command uplink schedule are needed 2 weeks (4 weeks during eclipse season) prior to final composition, approval and uplink. This allows time for SOCC to build and fully verify the commands. However, changes requiring only a few one-time commands can be performed within a matter of days and emergency commanding can be accomplished in minutes.

The SEC and AFWA forecast centers, both 24/7 operational units, have three areas of interest for SXI: development, training, and operations. Data products and software analysis tools for SEC and AFWA are developed and maintained by SEC (see Section 5 for detail). SEC provides training on data products, software, and the physics behind the SXI observations. Nominal operations using the data products and software tools are governed by operating procedures created and maintained by SEC and AFWA. These procedures include the maintenance, monitoring, and analysis of real-time image displays and movies; real-time, automated SXI flare event reports; forecaster-edited SXI flare event reports; operational region of interest definitions; and derived coronal hole boundaries.

The real-time availability goal for SXI data is $>95 \%$ with no outages longer than $72 \mathrm{~h}$. This includes major scenarios like facility-wide power failures, computer hardware crashes, and minor problems such as latency due to network loading. To support this goal, the data ingest and processing system itself is designed for unattended availability $>98.5 \%$ of the time, a National Centers for Environmental Prediction (NCEP, part of the National Weather Service) standard (National Centers for Environmental Prediction Year End Review, 2002). In addition, operational, 24/7 upkeep for the SEC ground data system and associated infrastructure is absolutely necessary and is provided by on-call support staff. During SXI operational periods the monthly on-time product generation has averaged $99.85 \%$, excluding part of the month of November 2003 when a software error limited accessibility to forecasters to $84 \%$. However, $99.99 \%$ of products were actually produced on time during that period.

Transitioning new products or software to the operational forecast center is a critical event for which risk is mitigated by maintaining a separate development and verification ground data system. All regression testing, user and maintenance documentation, training, and forecast operations procedures must be in place before the transition of a new product to the operational ground data system.

SEC and NGDC coordinate to ensure that metadata and images are delivered to the NGDC archive in near real-time. These data are immediately made available NGDC's SXI public web site, http://sxi.ngdc.noaa.gov. The delivery process, from image collection onboard GOES-12, to the web interface is less than 2 min under normal operating conditions. 
The SXI team at SEC performs long-term monitoring of data for changes in calibration, response, etc. These changes, along with changes in solar activity are used to modify the ground infrastructure, products, and/or the image sequences. In addition, SEC's SXI team researches and plans for new products. Development of new products requires occasional 'experimental' changes to operating sequences. Such sequence changes are carefully planned so as not to interfere with the operational objectives and mission of SXI. In addition, the SXI Team is the focal point for tracking and evaluation of both instrument and ground data system problems.

\subsection{Chronology OF OPERATIONS}

A summary of the operational history and the SXI's status during that history is a necessary component for selecting event periods for study. Though SXI was tested immediately after launch, it was placed into storage until GOES-8 was no longer able to maintain its orbit precisely enough for meteorological observations. After being reactivated, SXI experienced both minor and substantial degradations in performance. SXI periods of operation are summarized in Table V.

TABLE V

SXI operations chronology and database summary.

\begin{tabular}{|c|c|c|}
\hline Period & $\begin{array}{l}\text { Number of } \\
\text { images }^{\mathrm{a}}\end{array}$ & Description \\
\hline $\begin{array}{l}\text { Post-Launch Test (7 September- } \\
20 \text { December 2001) }\end{array}$ & 98719 & $\begin{array}{l}\text { Full instrument capability, majority of } \\
\text { images are high cadence patrol }\end{array}$ \\
\hline $\begin{array}{l}\text { GOES-12 on-orbit storage } \\
\text { (21 December-21 January 2003) }\end{array}$ & 0 & No SXI imaging \\
\hline $\begin{array}{l}\text { Pre-operational SXI testing } \\
\quad \text { (22 January-26 April 2003) }\end{array}$ & 131450 & $\begin{array}{l}\text { Pinhole light leak affecting 'Open' } \\
\text { filter band discovered } 22 \text { January } \\
2003\end{array}$ \\
\hline $\begin{array}{l}\text { Routine operations I (26 April- } \\
2 \text { September 2003) }\end{array}$ & 181893 & $\begin{array}{l}\text { Pinhole effect compensated in ground } \\
\text { data processing }\end{array}$ \\
\hline $\begin{array}{l}\text { HVPS anomaly investigation } \\
\quad(3 \text { September-28 October 2003) }\end{array}$ & $\sim 0$ & $\begin{array}{l}\text { No imaging except for low voltage } \\
\text { testing }\end{array}$ \\
\hline $\begin{array}{l}\text { Routine operations II ( } 28 \text { October- } \\
5 \text { November 2003) }\end{array}$ & 10263 & $\begin{array}{l}\text { Normal imaging with reduced } \\
\text { detector gain }\end{array}$ \\
\hline $\begin{array}{l}\text { Entrance filter failure analysis } \\
\quad(5-12 \text { November 2003) }\end{array}$ & $\sim 0$ & No solar imaging \\
\hline $\begin{array}{l}\text { Routine operations III (12 November } \\
\text { 2003-1 January 2005) }\end{array}$ & 588103 & $\begin{array}{l}\text { Operations with reduced detector gain } \\
\text { and loss of use of } 3 \mathrm{MK} \text { channel }\end{array}$ \\
\hline Total as of 1 January 2005 & 1010428 & \\
\hline
\end{tabular}

${ }^{a}$ Based on the NGDC SXI data archive at http://sxi.ngdc.noaa.gov. 
During Post-Launch Test (PLT) the SXI was fully exercised and initial designs for routine observations set. From 7 September to 18 October 2001, the SXI operated during weekdays roughly during working hours on the United States East Coast. While not performing specific tests, observations were conducted using a threefilter patrol sequence, consisting of seven exposures, which repeated every $7 \mathrm{~min}$. Starting 19 October, SXI observed continuously ( $24 \mathrm{~h}$ per day). On 7 November the sequence used when not conducting tests was changed to a different precursor of the SXI routine patrol sequence that used eight images in four filters repeated every $12 \mathrm{~min}$. While other minor changes to the 'routine' observing sequence were made, there is one final major period of interest with a fundamentally different observing scheme. Between 10 and 19 December the observing sequence run consisted of a single filter (Open) patrol at maximum cadence. Short - long exposure pairs were taken resulting in full-dynamic range coverage of the entire Sun at an effective cadence of $90 \mathrm{~s}$. A full listing of all SXI observing sequences conducted is available at http://sxi.ngdc.noaa.gov/sxi_data_notes.html.

After a year in on-orbit storage, GOES-12 was reactivated. During the first SXI high-voltage images of the Sun, taken on 22 January 2003, it became apparent that there was a contaminating light source when using the Open filter position. This offband 'light-leak' and its mitigation are described in detail in Paper 2. In summary, the problem was attributed to visible wavelength solar radiation penetrating somewhere near the front of the instrument, possibly through a pinhole in an entrance filter. Other analysis filters provided sufficient off-band rejection that the problem was not detectable. A micrometeorite hit on 19 January 2003 in the region of the satellite solar array may be related to the light leak. While specific tests were conducted during pre-operational testing, the vast majority of the time was spent in the routine observation mode described in Table IV. Upon formal entry into operations in 26 April 2003, the routine observation sequence shown in Table IV was utilized exclusively.

On 2 September 2003, the high-voltage system began to exhibit anomalous behavior that resulted in the instrument autonomously entering a safe mode. The SXI was shut down, except for a few diagnostic tests, until 28 October while an anomaly investigation was performed. No single cause for the failure could be clearly identified. Probable causes spanned the range of seriousness from a failed component in the HVPS telemetry system to an actual HV breakdown. Though additional testing might have resolved the cause, the possible consequence of losing all instrument functionality existed. Since the anomalous behavior only appeared to occur at or above $600 \mathrm{~V}$ and did not occur at or below $500 \mathrm{~V}$, it was decided that a safe return to operations, even with reduced gain was smarter than additional testing that might risk the instrument. SXI returned to operation on Oct 28, during the decay phase of a GOES X17 class flare. The only change to imaging operations was that the $\mathrm{MCP}$ was operated at a gain of $500 \mathrm{~V}$ for all solar images. Previously, imaging had been conducted with gains of 550-750 V. This change resulted in 
an effective decrease in SXI sensitivity by about $60 \%$. The sequence of images remained the same as in Table IV.

On 5 November, Open filter images began to appear dark with no image of the Sun. While they superficially resembled dark background images, details of the signal levels of the pixels were inconsistent. The SXI was commanded into safe mode while the situation was assessed. Fairly rapidly, it was determined that the Open position images were consistent with complete saturation of the CCD detector. Saturation occurred with no gain applied to the detector and for the shortest possible exposure time, $1.5 \mathrm{~ms}$. Simultaneously, low-level contaminating signals were seen in several other filter positions. These signals had the same general appearance as the previous Open position off-band light leak. Taken together, the most likely root cause is a complete failure of one of the six entrance filters. The visible light signal saturates the Open position, making it unusable. The contaminating signals seen in other filters are due to small pinholes illuminated by the visible Sun now through $\sim 1 / 6$ th of the entrance aperture. Paper 2 covers SXI performance and calibration up until this entrance filter failure. In addition to the loss of the utility of the Open filter, it was desired not to expose the CCD to direct sunlight. Because of the order of placement of the filters in the filter wheel, this precluded the use of the thick polyimide filter. A new observing sequence was designed and implemented on 12 November (Table VI). The thin polyimide filter was used as the primary patrol filter with long-exposure images taken every $2 \mathrm{~min}$. Short exposures in this filter are taken every $4 \mathrm{~min}$. Operating at reduced gain with the thin polyimide filter as the position with the greatest throughput has limited the ability of the SXI to study coronal holes, the quiet corona, and dynamic phenomena with lower contrast such as coronal waves and filament eruptions. However, SXI data remains useful for flare and active region studies.

TABLE VI

SXI Routine Observation Sequence Subsequent to 2003 Nov 12.

\begin{tabular}{|c|c|c|c|c|c|c|c|c|c|c|c|}
\hline \multirow{2}{*}{$\begin{array}{l}\text { Start } \\
\text { (UT) }\end{array}$} & \multirow{2}{*}{$\begin{array}{l}\text { Total } \\
\text { time }\end{array}$} & \multirow{2}{*}{$\begin{array}{l}\text { Number of } \\
\text { repetitions }\end{array}$} & \multirow{2}{*}{$\begin{array}{l}\text { Block } \\
\text { type }\end{array}$} & \multicolumn{8}{|c|}{ Minute number } \\
\hline & & & & 1 & 2 & 3 & 4 & 5 & 6 & 7 & 8 \\
\hline 01:00 & 8 & 1 & DEM & $\mathrm{Pn}_{\mathrm{S}}$ & $\mathrm{Pn}_{\mathrm{L}}$ & $\mathrm{LL}_{\mathrm{L}}$ & $\mathrm{Pn}_{\mathrm{L}}$ & $\mathrm{Pn}_{\mathrm{S}}$ & $\mathrm{Pn}_{\mathrm{L}}$ & $\mathrm{Bm}_{\mathrm{L}}$ & $\mathrm{Pn}_{\mathrm{L}}$ \\
\hline 01:09 & 112 & 14 & Patrol & $\mathrm{Pn}_{\mathrm{S}}$ & $P n_{L}$ & $\mathrm{Pm}_{\mathrm{L}}$ & $\mathrm{Pn}_{\mathrm{L}}$ & $\mathrm{Pn}_{\mathrm{S}}$ & $\mathrm{Pn}_{\mathrm{L}}$ & $B n_{L}$ & $P n_{L}$ \\
\hline 03:00 & 8 & 1 & $\mathrm{Bkg}$ & $\mathrm{Pn}_{\mathrm{S}}$ & $P n_{L}$ & $\operatorname{Pn}_{X L}$ & $\mathrm{LL}_{X \mathrm{~L}}$ & $\mathrm{Pn}_{\mathrm{S}}$ & $\mathrm{Pn}_{\mathrm{L}}$ & $\mathrm{Bg}_{\mathrm{S}}$ & $\mathrm{Bg}_{\mathrm{L}}$ \\
\hline 03:09 & 112 & 14 & Patrol & $\mathrm{Pn}_{\mathrm{S}}$ & $P n_{L}$ & $\mathrm{Pm}_{\mathrm{L}}$ & $P n_{L}$ & $\mathrm{Pn}_{\mathrm{S}}$ & $P n_{L}$ & $B n_{L}$ & $P n_{L}$ \\
\hline 05:00 & 8 & 1 & $\mathrm{Bkg}$ & $\mathrm{Pn}_{\mathrm{S}}$ & $P n_{L}$ & $\operatorname{Pn}_{X L}$ & $\mathrm{LL}_{X L}$ & $\mathrm{Pn}_{\mathrm{S}}$ & $\mathrm{Pn}_{\mathrm{L}}$ & $\mathrm{Bg}_{\mathrm{S}}$ & $\mathrm{Bg}_{\mathrm{L}}$ \\
\hline 05:09 & 112 & 14 & Patrol & $\mathrm{Pn}_{\mathrm{S}}$ & $\mathrm{Pn}_{\mathrm{L}}$ & $\mathrm{Pm}_{\mathrm{L}}$ & $\mathrm{Pn}_{\mathrm{L}}$ & $\mathrm{Pn}_{\mathrm{S}}$ & $\mathrm{Pn}_{\mathrm{L}}$ & $B n_{L}$ & $\mathrm{Pn}_{\mathrm{L}}$ \\
\hline
\end{tabular}

Key: Pn, thin polyimide; Pm, medium polyimide; Bn, thin beryllium; Bm: medium beryllium; Bg, background (dark) frame; LL, light-leak background (thin polyimide filter). Subscripts: S, 'short' exposure (<1 s); L, 'long' exposure (3 s); XL, 'extra-long' exposure (12 s). 


\section{Data Processing and Distribution}

Like instrument and mission operations, data processing and distribution for the SXI is driven by SXI's operational role. This requires an emphasis on near real-time generation and distribution of high-quality image data and derived products at a very high availability. Therefore, SXI telemetry is downlinked directly to SEC in Boulder, Colorado. This data stream is assembled into images, processed to remove defects, and used to generate products for forecasters. Our goal is to provide $>98.5 \%$ (on a monthly basis) of processed image data to forecaster workstations within $100 \mathrm{~s}$ of exposure completion at the spacecraft. In addition, the images and products are archived by NOAA's NGDC, co-located in Boulder with SEC, to preserve the historical record of solar activity and to allow researchers to improve forecasting models. NGDC generally makes SXI data available at its SXI web site within 2 min of exposure completion.

\subsection{REAL-TIME DATA PROCESSING AND DISTRIBUTION}

SEC's ground data system implemented for the SXI provides a robust conduit for data and products with significant redundancy and graceful degradation/recovery in case of systems failures. The system is depicted in Figure 6.

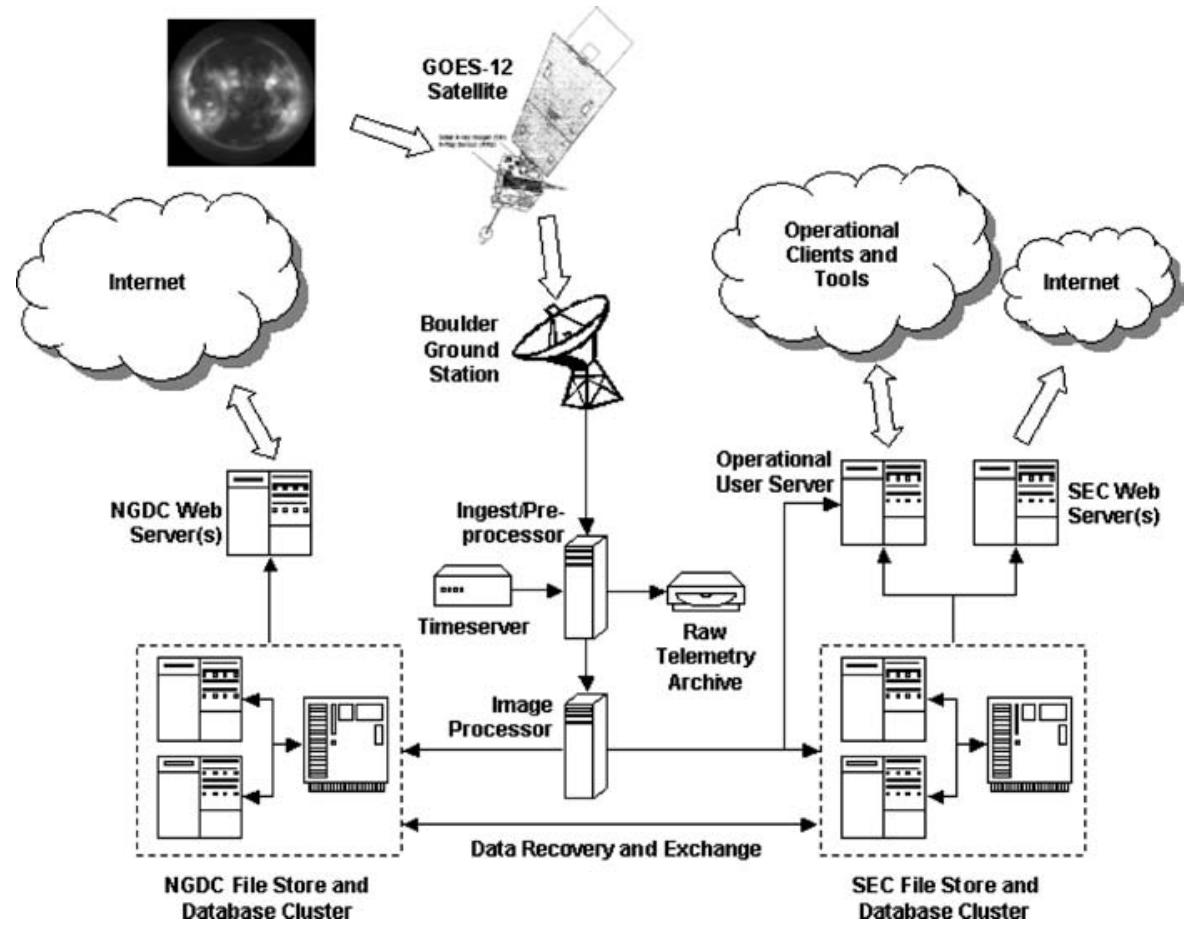

Figure 6. Ground data system. 
SXI telemetry data received at the NOAA Boulder ground station is assembled into raw (Level-0) image files by the ingest and preprocessing system. A network timeserver supports time tagging of data. Parity checking is performed to enable single bit error corrections. This real-time process also generates other housekeeping and status products as ASCII format files. Both types of files are transferred to a circular file buffer on the image-processing platform for further processing and distribution. The raw telemetry bit stream is archived to DVD. This raw telemetry stream can be replayed on an independent processing system for the purpose of data recovery or product improvements.

The image processor generates cleaned and calibrated (Level-1) images, archives metadata in a relational database, archives image files on a mass storage system, and forwards images to the real-time operational server. In addition, it produces such derived data products as lists of X-ray bright regions on the Sun. This processor is composed of control code that oversees a set of Interactive Data Language (IDL) algorithms and provides the interfaces to other elements of the ground data system. The IDL algorithms take advantage of the SolarSoft Libraries. While the operational IDL code remains under rigorous configuration management internal to SEC, a version of it is made available through SolarSoft.

SXI images are stored as SolarSoft compliant FITS files on a Redundant Array of Inexpensive Disks (RAID) system. A relational database is used to index and track the image files and contains metadata about images. SEC maintains an operational store of 60 days worth of image files, but stores the metadata indefinitely. NGDC receives data from SEC in real time and archives all images and metadata indefinitely. This includes offsite data copies for security.

Operational users of SXI data include NOAA, USAF, and NASA (Section 4.2). Distribution of the SXI products to these users is done using Common Object Request Broker Architecture (CORBA). A server provides access to all real-time and archived products to registered clients over both the local intranet and the global Internet. This server receives products in near real-time from the image processor and provides rapid and flexible access to the operational database/file server for archived products.

For in-depth analyses, forecasters and other operational users need quantitative analysis and visualization tools. As part of the ground data system, SEC has developed the Image Display and Analysis Client (IDAC) for quantifying solar features. It has also developed the flexible Movie Display and Analysis Client (MDAC) player for visualization and assessment of dynamic changes in the corona. Both of these tools were developed in IDL and take advantage of SolarSoft.

\subsection{SXI ON THE INTERNET}

The SXI program maintains a presence on the Internet to provide researchers, educators, the public, and other non-operational users with data as well as instrument performance, status, and programmatic information. This web presence 
consists of the closely linked sites of SEC (http://www.sec.noaa.gov/sxi) and NGDC (http://sxi.ngdc.noaa.gov). Along with latest images, movies and news, the SEC site provides a detailed Science User Guide for researchers using SXI data. The user guide provides detailed and up to date information on SXI instrument performance, data products, and software. In addition, the SEC site contains information on future SXI instruments, including the GOES N/O instruments with an anticipated first launch in 2005, and the instrument planning activities for 2012 and beyond.

As the archivist of SXI data, NGDC is the source of data for the public. The NGDC web site provides some background information on the SXI, but its main task is the selection and delivery of SXI data. The initial default display shows the most recent sequence of images as a table of thumbnail images. Users may browse these images then select data into a 'shopping cart' for download. The browse capability is flexible and allows users to step though the archive with a programmable interval, i.e., $1 \mathrm{~min}$, daily, solar rotation, etc. Alternatively, data may be displayed in a 'matrix' format with columns of fixed image type, e.g., 'coronal structure', and time advancing down the page.

A significant search capability is built into the NGDC web site including the ability to search by wavelength, exposure duration, time interval, image type, detector gain, etc. Both Level-0 and Level-1 images are available for retrieval and delivery. Once a selection has been made, the files are compressed. An e-mail message provides the requester with a URL for downloading the compressed files. Up to $500 \mathrm{MB}$ of data ( $\sim 500$ Level-1 images) can be downloaded at once. Alternatively, NGDC provides direct HTTP access to SXI image files via IDL routines available in SolarSoft. A catalog summary function on the web site shows SXI coverage periods.

Finally, NGDC uses the image metadata to create static movie loops and updating movie loops in MPEG format. Updating movies include 12-h and 54 day sequences. Static movies include 1-day and 1-month versions.

\subsection{SOLARSOFT}

While predominantly an operational instrument, the research potential of SXI data is significant. The SXI program facilitates the use of its data by providing a software branch ( /SSW/GOES/SXIG12) to the SolarSoft Library of IDL routines (Freeland and Handy, 1998). There are four key programs for research users:

SXI_FILES/SXI_COPY - Directly downloads (via sockets) SXI data files at the NGDC archive or a local file repository.

SXIG12_PREP - Processes raw (Level-0) FITS files into background subtracted, flat-fielded, exposure normalized (Level-1) images. This code is updated such that it recognizes changes in SXI calibration (for which corrections exist) based on the date of the data to be calibrated. Thus, it will automatically apply an Open 
position light-leak correction for Open filter data after 22 January 2003. Note that Level-1 FITS data between 22 January and 24 April 2003 in the NGDC archive do not have this correction applied. Changes in response due to the entrance filter failure on 5 November 2003 have not be quantified and are thus not included in this calibration routine.

IDAC - Image display and analysis tool allowing quantitative analysis of SXI images with such features as zoom, profiling image slices, region statistics, etc.

MDAC - Movie display and analysis tool that uses SXI browse images for quantitative analysis of dynamic events with such features as zoom, running differences, fixed/base differences, etc.

Software existing in operational form, but not yet prepared for a public distribution include: point spread function deconvolution and automated bright region location. Efforts are beginning to support analysis of temperature and emission measure, DEM, and solar soft X-ray irradiance. While resources of the SXI team are limited, our intent is to work jointly with the research community to further develop advanced analysis capabilities for SXI data.

\section{Summary}

The GOES-12 SXI provides a new, operational capability for space weather forecasters. In addition, it opens a new window on the solar corona, in cadence, spectral coverage, and continuity. The performance details provided in Paper 2 cover the period prior to 5 November 2003, when a performance-degrading failure occurred. This period contains a wealth of data in over 420000 images of the Sun.

Necessary accommodations have been made in the design and operation of the instrument to facilitate hosting on a nadir pointing weather satellite. The very stable and accurate pointing usually associated with a solar imaging instrument was traded for accurate pointing knowledge. This allowed the economical deployment of the SXI on an operational spacecraft in an orbit that provides for unprecedented continuity of observations while simultaneously supporting a data rate required for high observing cadence.

The SXI optics are rather traditional Wolter I grazing incidence optics. However, the unique application of MCP-intensified CCD detector allows the SXI to take images in a previously sparsely sampled region of the solar spectrum. This spectral region contains emissions bridging the coronal temperature regimes observed by the SOHO EIT instrument and the Yohkoh SXT instrument. Combined with its high cadence, synoptic observing program, SXI data complement many other scientific observations of the Sun.

SXI operations and data distribution are all driven by the need to supply calibrated data to space weather forecasters in real-time. As an ancillary benefit, SXI data are made available in near real-time to the research community via the Internet. This availability and timeliness has made SXI data useful for the Big Bear Solar 
Observatory Active Region Monitor (ARM) (Gallagher, Moon, and Wang, 2002) and the SolarSoft 'Latest Events' web sites ${ }^{1}$ as well as the automated Max Millenium Program ${ }^{2}$ flare catalog.

The major performance degradation that occurred in 2003 is due to the loss of one of the six entrance pre-filters on the instrument. This loss rendered the Open filter position unusable due to saturation from solar white light and the primary imaging filter used now is the denser, PTHN filter. The thin polyimide filter is sensitive to slightly hotter solar plasma temperatures than the OPEN position. Due to constraints on the detector gain settings, the signal-to-noise level in typical SXI patrol images has decreased as well. However, the instrument remains very useful for the study of active regions and flares. In December 2004, SEC deployed a new image product constructed by stacking multiple images, which substantially improves signal-to-noise. This enables the recovery of the ability to determine coronal hole boundaries, which is one of the main SXI objectives. Finally, sounding rocket underflight calibration launches are planned for GOES X-ray instruments, including the SXI, in 2004 and 2006. A future publication will document the results of these flights and the performance of the SXI in its post filter-failure state.

As the first in a series of solar X-ray imagers on GOES spacecraft, the GOES-12 SXI serves as the prototype for demonstrating the delivery of operational X-ray image products both to space weather forecasters and to the research community. The GOES-12 SXI is unique. The next block of SXIs, to be launched starting in 2004, will have a substantially different design and are expected to provide higher spatial resolution and sensitivity. And finally, the planning stages are beginning for the block of advanced SXIs to follow in 2012.

\section{Acknowledgments}

We and NOAA would like to express appreciation for the financial support of USAF that was necessary to bring this project to flight. Additionally, in-kind support from NASA Marshall Space Flight Center aided in making this project a reality.

\section{References}

GOES I-M Data Book: 1996, NASA Goddard Space Flight Center, Greenbelt, MD, U.S.A., p. 196. National Centers for Environmental Prediction Year End Review, pp. 18, National Centers for Environmental Prediction, Camp Springs, Maryland, 2002.

Bornmann, P. L., Speich, D., Hirman, J., Matheson, L., Grubb, R., Garcia, H., and Viereck, R.: 1996a, Proceeding of SPIE on GOES-8 and Beyond, Vol. 2812, pp. 291-298.

Bornmann, P. L., Speich, D., Hirman, J., Pizzo, V., Grubb, R. N., Balch, C., and Heckman, G.: 1996b, in Proceedings of SPIE on GOES-8 and Beyond, Vol. 2812, pp. 309-319.

${ }^{1}$ http://beauty.nascom.nasa.gov/arm/latest/; http://www.lmsal.com/solarsoft/latest_events/

${ }^{2}$ http://mithra.physics.montana.edu/max_millennium/ 
Bornmann, P. L., Pizzo, V. J., Speich, D., Cauffman, S., Hooker, R., Russell, K., Wallace, S., Davis, J., Buschmann, S., and Beranek, R.: 1997, AAS Solar Physics Division Meeting.

Cessna, J. R., Hoover, R. B., Grubb, R. N., Orswell, P. L., and Taylor, J. H.: 1983, The GOES X-ray Imager Feasibility Demonstration, NOAA/SEL, Boulder, Colorado, p. 83.

Corder, E. L.: 1994, Proceedings of SPIE on Space Instrumentation and Dual-Use Technology, Vol. 2214, 294.

Davis, J., Bagdigian, D., Buschmann, S., Russell, K., and Wallace, K.: 1994, AIAA Space Programs and Technologies Conference, Huntsville, AL, U.S.A.

Delaboudiniere, J.-P., Artzner, G. E., Brunaud, J., Gabriel, A. H., Hochedez, J. F., Millier, F., Song, X. Y., Au, B., Dere, K. P., Howard, R. A., Kreplin, R., Michels, D. J., Moses, J. D., Defise, J. M., Jamar, C., Rochus, P., Chauvineau, J. P., Marioge, J. P., Catura, R. C., Lemen, J. R., Shing, L., Stern, R. A., Gurman, J. B., Neupert, W. M., Maucherat, A., Clette, F., Cugnon, P., and van Dessel, E. L.: 1995, Solar Phys. 162, 291.

Freeland, S. L. and Handy, B. N.: 1998, Solar Phys. 182, 497.

Gallagher, P. T., Moon, Y.-J., and Wang, H.: 2002, Solar Phys. 209, 171.

Krieger, A., Paolini, F., Vaiana, G. S., and Webb, D.: 1972, Solar Phys. 22, 150.

Martens, P. C. H. and Cauffman, D.: 2002, Multi-Wavelength Observations of Coronal Structure and Dynamics: Yohkoh 10th Anniversary Meeting, Elsevier.

Mewe, R., Gronenschild, E. H. B. M., and Oord, G. H. J. v. d.: 1985, Astron. Astrophys. Suppl. Ser. 62, 197.

Onsager, T., Grubb, R., Kunches, J., Matheson, L., Speich, D., Zwickl, R. W., and Sauer, H.: 1996, Proceedings of SPIE on GOES-8 and Beyond, 2812, 281.

Poppe, B. B.: 2000, EOS Transactions, AGU, p. 322.

Powell, F. R., Keski-Kuha, R. A., Zombeck, M. V., Goddard, R. E., Chartas, G., Townsley, L. K., Moebius, E., Davis, J. M., and Mason, G. M.: 1997, Proceedings of SPIE on Grazing Incidence and Multilayer X-Ray Optical Systems, 3113, 432.

Russell, K., Briscoe, J., Corder, E., Wallace, S., and Chappell, J. H.: 1996, Proceedings of SPIE on GOES-8 and Beyond, 2812, 638.

Singer, H., Matheson, L., Grubb, R.N., A., and Bouwer, D.: 1996, Proceedings of SPIE on GOES-8 and Beyond, 2812, 299.

Smithers, M. E. and Zissa, D. E.: 1996, SPIE 2805, 115.

Tsuneta, S., Acton, L., Bruner, M., Lemen, J., Brown, W., Caravalho, R., Catura, R., Freeland, S., Jurcevich, B., and Owens, J.: 1991, Solar Phys. 136, 37.

Underwood, J. H. and McKenzie, D. L.: 1978, Solar Phys. 60, 311.

Vaiana, G. S. and Rosner, R.: 1978, Annu. Rev. Astron. Astrophys. 16, 393.

Vaiana, G. S., Krieger, A. S., and Timothy, A. F.: 1973, Solar Phys. 32, 81.

Wallace, K. S. and Freestone, K.: 2001, Solar X-Ray Imager (SXI) Operators Handbook, NASA Marshall Space Flight Center, Huntsville, Alabama, p. 497.

Wallace, K. S., Brown, T. A., and Freestone, K. A.: 1998, 17th Digital Avioncis Conference, Seattle, Washington.

Watanabe, T., Kosugi, T., and Sterling, A. C.: 1998, Observational Plasma Astrophysics: Five Years of Yohkoh and Beyond, Klewer Academic Publishers, Dordrecht, Boston, MA.

Zimmermann, F., Zwirn, G., and Davis, M. A.: 2004, Proceedings of SPIE on Telescopes and Instrumentation for Solar Astrophysics, 5171, p. 326. 